\title{
A closed core microfluxgate sensor with cascaded planar FeNi rings
}

\author{
O. Zorlu ${ }^{\mathrm{a}, \mathrm{b}, *}$, P. Kejik ${ }^{\mathrm{b}}$, W. Teppan ${ }^{\mathrm{c}}$ \\ a Middle East Technical University, MEMS Center, Ankara, Turkey \\ ${ }^{\mathrm{b}}$ Ecole Polytechnique Fédérale de Lausanne (EPFL), IMT, Lausanne, Switzerland \\ ${ }^{c}$ LEM, Geneva, Switzerland
}

\section{A R T I C L E I N F O}

\section{Article history:}

Received 30 September 2009

Received in revised form 19 January 2010

Accepted 19 February 2010

Available online 3 March 2010

\section{Keywords:}

Magnetic sensors

Fluxgate

Ring-type microfluxgate

Closed magnetic loop

Electroplated FeNi

Linear operation range

\begin{abstract}
A B S T R A C T
In this paper, we present a new microfabricated fluxgate sensor structure using cascaded planar rings as the ferromagnetic core. The planar ring structures provide closed magnetic excitation loops enabling uniform core saturation with relatively small excitation magnetic field. The magnetic excitation is provided with a rod passing through the ring cores. Planar coils placed under the edges of the core are used as sensing elements. By using this structure, fluxgate sensors having a closed core configuration are realized with a developed microfabrication process in a very small area $\left(<0.1 \mathrm{~mm}^{2}\right)$ with reduced number of via connections. The ferromagnetic rings are realized with a standard FeNi (iron-nickel) electroplating process. The main advantage of this structure is the possibility to arrange the linear operation range of the sensor by only changing the number of ring cores, without affecting the excitation mechanism. This is demonstrated by simulations and microfabricated prototypes having 18 and 12 ring cores with $\pm 300 \mu \mathrm{T}$ and $\pm 550 \mu \mathrm{T}$ linear operation ranges, respectively. A maximum linear operation range of $\pm 2 \mathrm{mT}$ is achieved with a larger size, 4-ring core. This is the widest linear operation range achieved with the microfabricated fluxgate type sensors, without using a feedback loop, to the best of our knowledge.
\end{abstract}

(C) 2010 Elsevier B.V. All rights reserved.

\section{Introduction}

Fluxgate type magnetic sensors are used in the magnitudal and directional measurement of DC or low-frequency AC magnetic fields. High linearity, high temperature stability, and low offset characteristics of fluxgate sensors enable their use in various fields. Fluxgate sensors are typically used in electronic compasses, current sensors, magnetic ink reading, detection of ferrous materials, and non-destructive testing [1]. The operation principle of a fluxgate sensor depends on the periodic saturation of a ferromagnetic material with an excitation magnetic field, mostly provided by an AC current, and detecting the change in the magnetic flux through the ferromagnetic core in the presence of an external magnetic field to be measured. Planar or solenoidal coils are used for the signal detection, where the 2 nd and higher order even harmonics of the induced coil voltage has the information about the external magnetic field.

Most of the millimeter sized fluxgate sensors such as PCB implemented ones use the closed ferromagnetic core configuration with race-track or toroidal core geometries [2,3]. By using these geometries, the ferromagnetic core of the sensor can be saturated uniformly and with smaller excitation field due to the closed mag-

\footnotetext{
* Corresponding author at: Middle East Technical University, MEMS Center, Ankara, Turkey. Tel.: +90 3122104546.

E-mail address: zorlu@metu.edu.tr (O. Zorlu).
}

netic loop for the excitation field formed around the core. On the other hand, in the case of integrated fluxgate sensors, open core [4] or almost closed core [5] geometries are preferred since they are more easily adapted to microfabrication technologies. However, the open core configuration forces the use of special magnetic films with very low coercivity values in order to be able to saturate them with small excitation field values [6]. This introduces non-standard steps to the microfabrication process flow. There are a few microfabricated closed core fluxgate examples available in the literature which use conventional thin film technologies such as sputtering, evaporation and electroplating $[7,8]$. On the other hand, these sensors utilize solenoidal coils both for excitation and detection, complicating the fabrication process, increasing the sensor area due to the large number of required via connections, and increasing the power dissipation of the sensor.

In this paper, we present a new microfluxgate sensor structure using cascaded planar ring cores that can be saturated with a single excitation rod passing through the middle of the ring structures. The presented sensor structure decreases the number of required vias, reducing the required sensor area and the resistance of the excitation rod, hence the power dissipation [9]. The closed core configuration is maintained with the ring shaped cores. A single ring core can be realized in a relatively small area and required saturation field can be provided with a relatively small excitation current. The ferromagnetic core of the sensor is fabricated by using standard FeNi electroplating process. Furthermore, the sensitivity and the linear operation range of the sensor can be varied by chang- 
ing the number of cascaded ring cores. The output signal is provided by planar sensing coils placed under the edges of the cascade.

\section{Operation principle, sensor structure, and design}

Fig. 1 presents the basic fluxgate operation principle. The operation principle is based on the periodic saturation of a ferromagnetic core material with an alternating excitation field, where the ferromagnetic core resides in an external magnetic field to be measured. The permeability of the ferromagnetic core is modulated according to its $B-H$ curve (Fig. $1(\mathrm{a})$ ), between $\mu_{0} \mu_{\mathrm{r}}$ and $\mu_{0}$ with the periodic excitation field at a certain frequency. When the material is not saturated, it operates in the linear region of its $B-H$ curve where it has a high permeability which is equal to $\mu_{0} \mu_{\mathrm{r}}$. Accordingly, the magnetic flux due to the external field to be measured is concentrated inside the core (Fig. 1(b)). When it is saturated, the permeability of the material drops to $\mu_{0}$, and magnetic flux passing thorough the material is chopped (Fig. 1(c)). This occurs twice in each period of the excitation field, so the external magnetic field information can be seen at the second (and higher even numbered) harmonic components of the power spectrum of the total flux or the change of flux with time (Fig. 1(d)).

Fig. 2 illustrates a single planar ring core (a) and the cascaded fluxgate sensor structure (b) presented in this paper. An alternating excitation current passing through the excitation rod creates a periodic circular magnetic field on the ferromagnetic ring (Fig. 2(a)). The peak value of this current is high enough to create a magnetic field required for the saturation of the ferromagnetic core. The external magnetic field information is extracted from the induced voltage across the planar sensing coil placed under the edges of the cascaded cores. The sensor is formed by cascading the planar ring cores, which are composed of a ferromagnetic ring and a coaxial excitation rod passing through the core. This single planar ring core structure can also be used as a fluxgate sensor core as in the case of a parallel ring-type fluxgate [2]. However, the structure has a

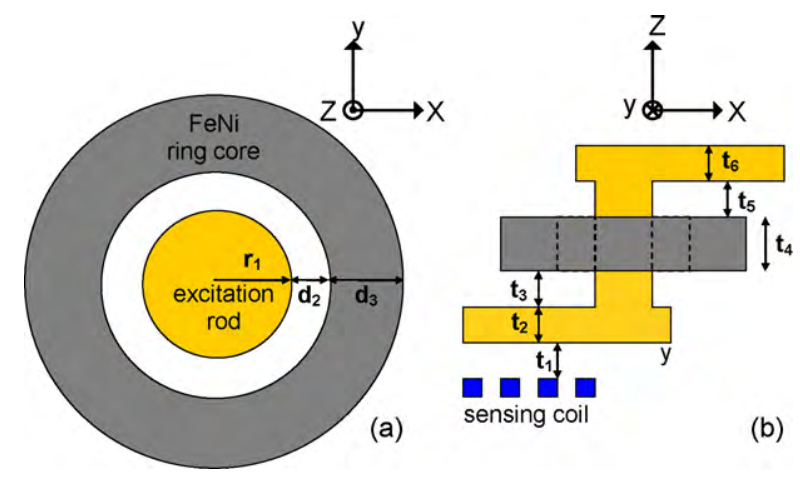

Fig. 3. Top view (a) and the side view (b) of the single ring structure with labeled dimensions.

very high demagnetization factor in the sensing direction when its dimensions are scaled down for microfabrication, which results in a very low sensitivity value. Because of this, several rings overlapping each other are cascaded as shown in Fig. 1(b). This provides a lower demagnetization factor along the length of the cascade of the rings, resulting in a higher sensitivity. Furthermore, the linear operation range of the sensor can be arranged by changing the number of rings in the cascade without affecting the excitation mechanism.

The dimensions of the single ring core and the number of rings to be used in the cascade are designed by using Maxwell ${ }^{\circledR}$ 3D FEM (Finite Element Modelling) tool. Fig. 3 shows the distances used as design parameters for the single ring core. The simulated parameters are the required peak excitation current and the linear operation range and sensitivity of the cascaded structure. The required peak value of the current for saturating the ferromagnetic core is determined by the total radius of the ring structure $\left(r_{1}+d_{2}+d_{3}\right)$ as illustrated in Fig. 3(a). Fig. 4 shows the simulation results for different ring structures with $r_{1}$ and $d_{2}$ are fixed to $3 \mu \mathrm{m}$
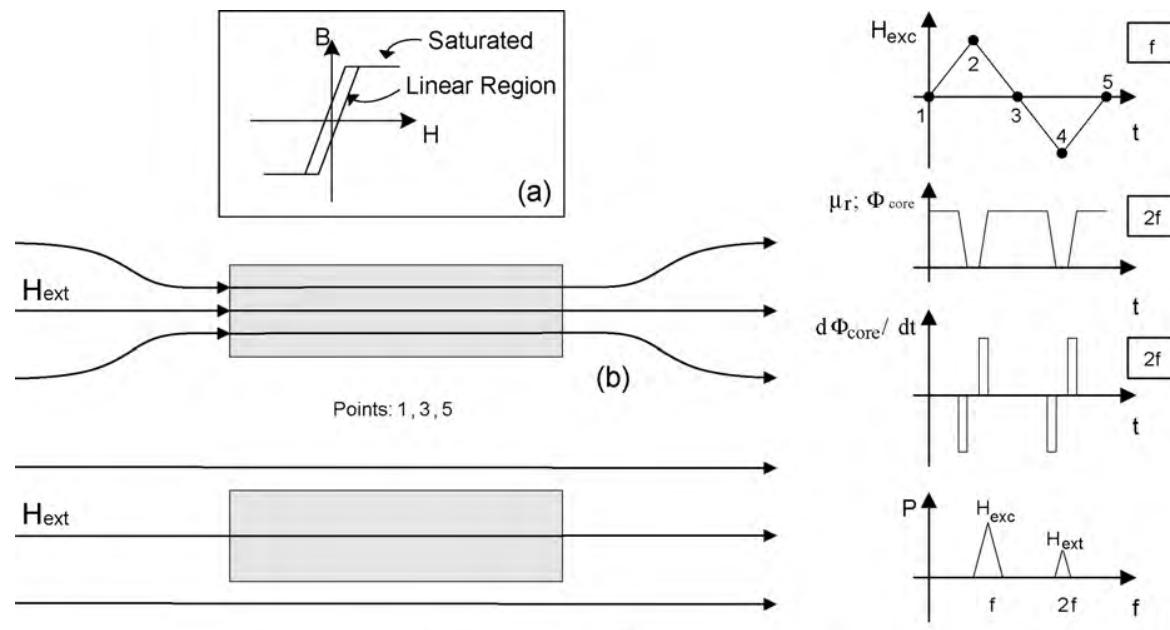

Points: 2,4

(c)

Fig. 1. The basic fluxgate operation principle.
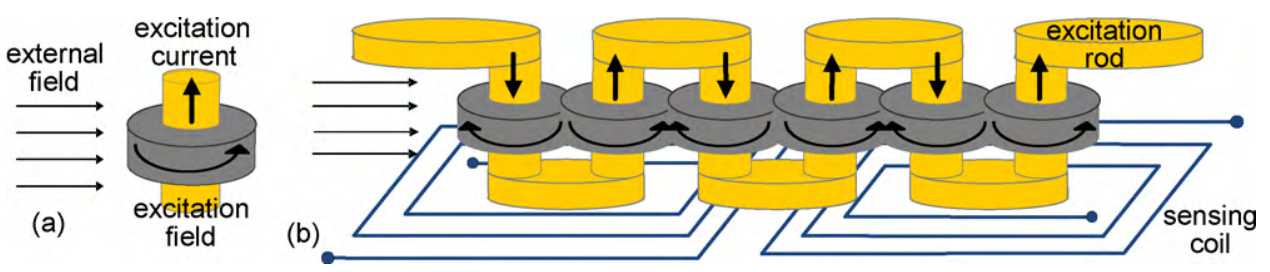

Fig. 2. (a) A single planar ring core and (b) cascaded fluxgate sensor structure. 


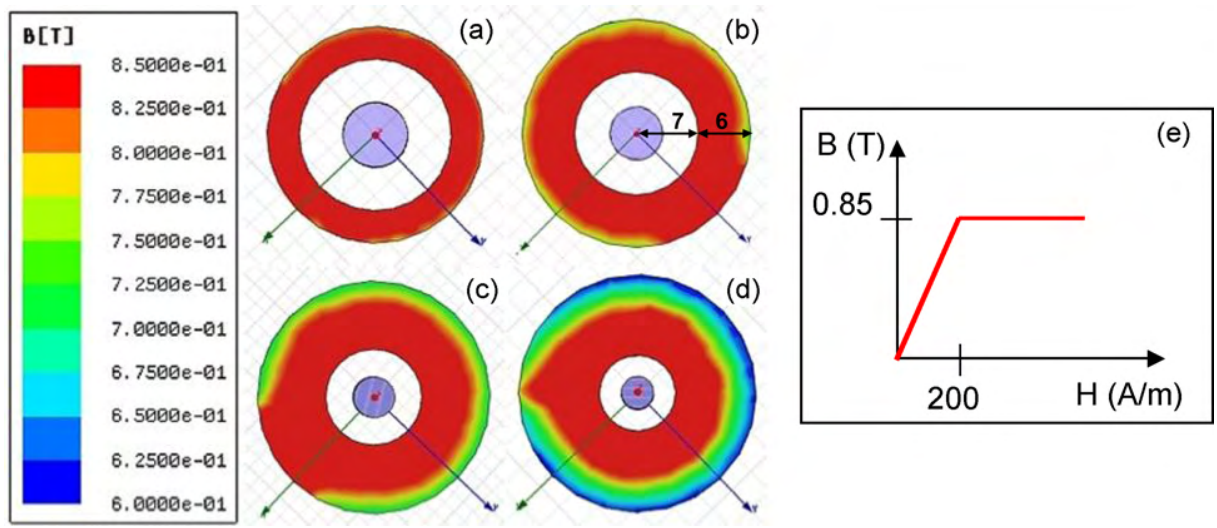

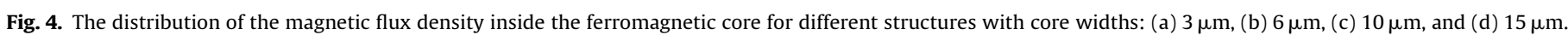
The $r_{1}$ and $d_{2}$ values are fixed to $3 \mu \mathrm{m}$ and $4 \mu \mathrm{m}$, respectively; (e) the magnetization curve used for the simulations.

and $4 \mu \mathrm{m}$, respectively, while $d_{3}$ is varied between $3,6,10$, and $15 \mu \mathrm{m}$. The core thickness $\left(t_{4}\right)$ is set to $2 \mu \mathrm{m}$. The $B-H$ curve of the simulated ferromagnetic material is also presented in Fig. 4. The current passing through the excitation rod is $20 \mathrm{~mA}$. The plotted value is the magnitude of the magnetic flux density on the cutplane at the middle of the ferromagnetic ring. As the radius of the structure increases, the outer edges of the core require more current for saturation. According to the simulation results, it is possible to saturate a structure having a $6 \mu \mathrm{m}$ wide ferromagnetic core with a current slightly higher than $20 \mathrm{~mA}$.

Fig. 5 shows the simulation results presenting the effect of varying the number of rings in the cascade, on the linear operation range and sensitivity of the sensor. A single ring structure has $6 \mu \mathrm{m}$ core width and $2 \mu \mathrm{m}$ core thickness. The relative permeability $\mu_{\mathrm{r}}$ of the ferromagnetic material is taken as 10,000 . For this simulation, the flux passing through the coil plane ( $z$-component of $B$ over $z=-\left(t_{1}+t_{2}+t_{3}\right)$ plane) under the ring structure is calculated by using (1) for each applied external magnetic field value. It should be noted that half of the plane under one edge of the core is taken into consideration as the magnetic field distribution over the other half plane is symmetrical with anti-symmetrical direction.

$$
\begin{aligned}
\left.\Phi_{x y}\right|_{z=-\left(t_{1}+t_{2}+t_{3}\right)} & =\int \vec{B} \cdot \overrightarrow{d A}=\left.\iint_{x y} B_{z} d y d x\right|_{z=-\left(t_{1}+t_{2}+t_{3}\right)} \\
& =\int_{x=0}^{x_{i}} \int_{y=0}^{y_{j}} B_{z} d y d x=\sum_{i=0}^{x_{i}} \sum_{j=0}^{y_{j}} B_{z}(i, j) \times \Delta x \Delta y
\end{aligned}
$$
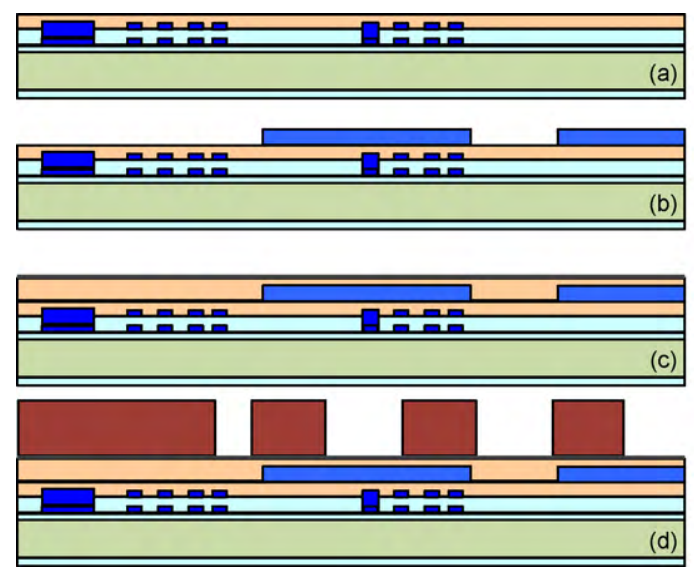

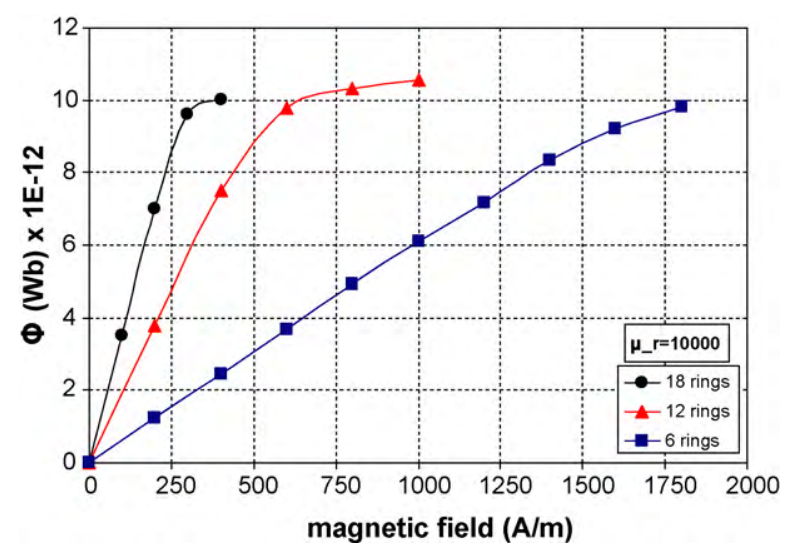

Fig. 5. The change of the flux with the applied magnetic field for cores having different number of rings.

The increase in the linear operation range with decreasing number of rings due to the increasing demagnetization effect is verified with the simulation results. It is seen from Fig. 5 that the linear range increases, however, the sensitivity decreases rapidly for reduced number of cascaded rings.

\section{Fabrication}

Fig. 6 shows the fabrication process flow that is developed for realizing the presented sensor structures. Silicon wafers with
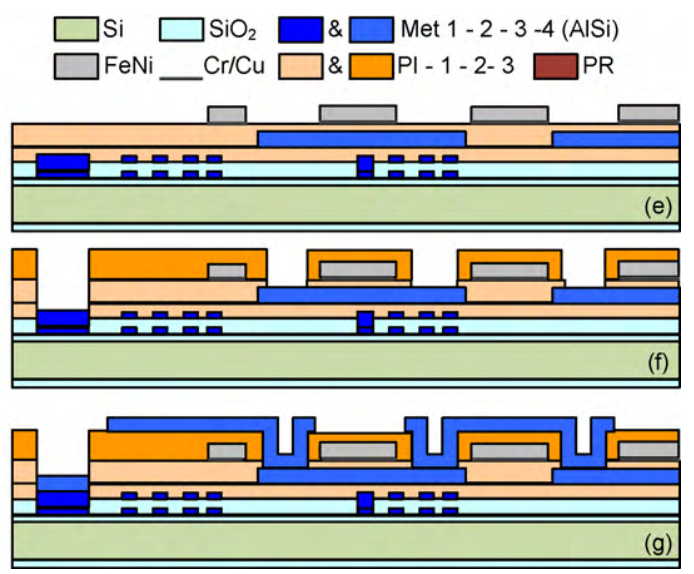

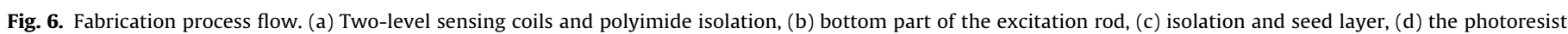
mold, (e) FeNi electroplating, (f) isolation and via opening, and (f) top of the excitation rod. 


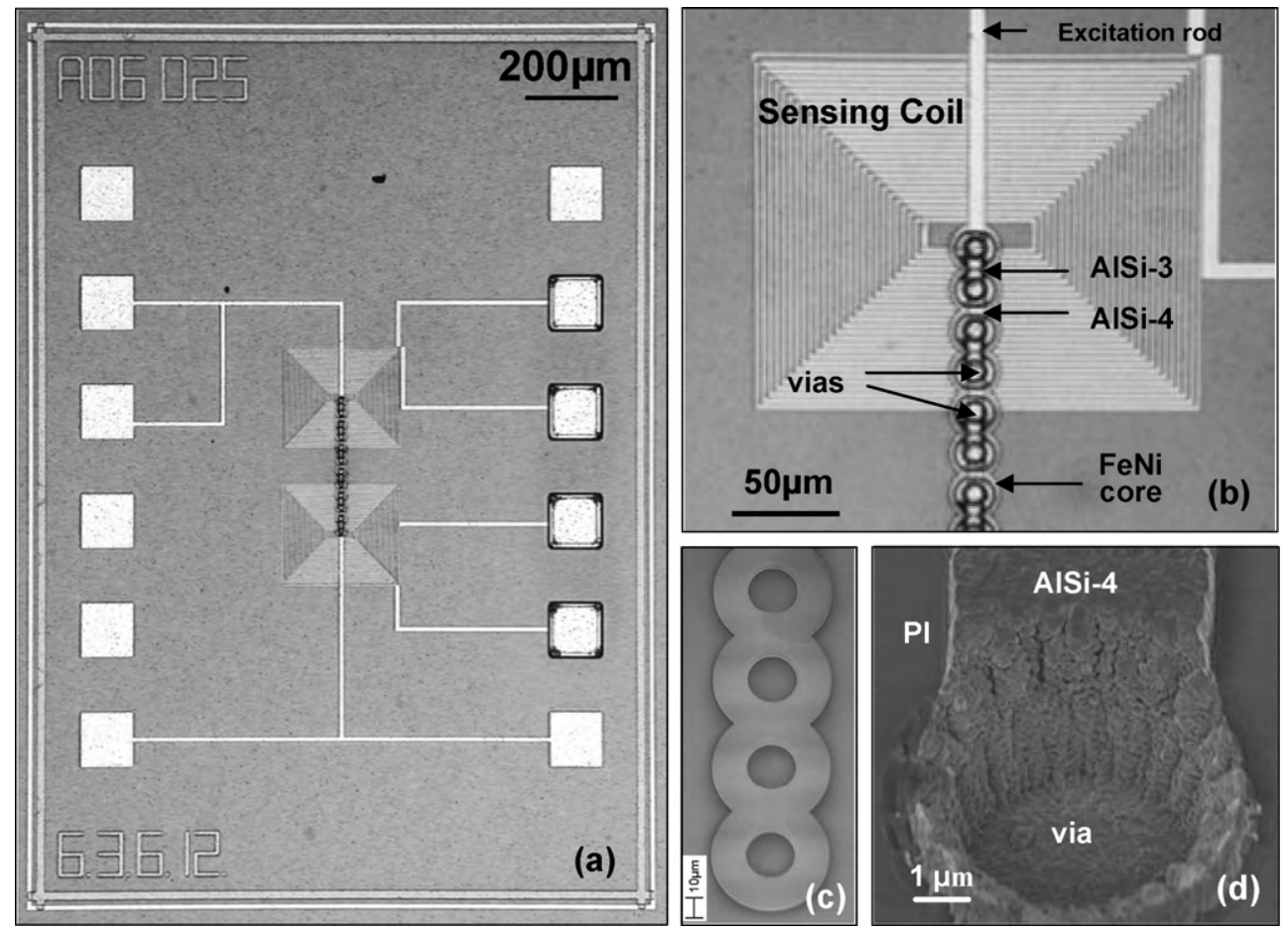

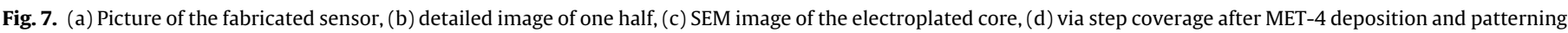

$0.5 \mu \mathrm{m}$-thick $\mathrm{SiO}_{2}$ layer are used as substrates. Firstly, two-level $0.5 \mu \mathrm{m}$-thick AlSi sensing coils separated by a $0.8 \mu \mathrm{m}$-thick $\mathrm{SiO}_{2}$ layer are formed on the substrate by using sputtering and RIE etching. A passivation layer is formed on the coils by spinning a $1.5 \mu \mathrm{m}$-thick polyimide layer over the coils (Fig. 6(a)). After this step, the 3rd AlSi layer is deposited and patterned over the polyimide layer (Fig. 6(b)). This $2 \mu \mathrm{m}$-thick layer serves as the bottom part of the excitation rod.

The 2 nd polyimide layer, which is $2.5 \mu \mathrm{m}$-thick is spun over the 3rd metal layer, providing an electrically isolated and planar surface for the next steps. Then, a $20 \mathrm{~nm} / 200 \mathrm{~nm} \mathrm{Cr} / \mathrm{Cu}$ seed layer is evaporated on the surface as the seed layer for the ferromagnetic core electroplating step. An AZ9260 photoresist mold is provided for electroplating the ferromagnetic ring core structures (Fig. 6(c)). Then, a $2 \mu \mathrm{m}$-thick FeNi layer is electroplated into the openings of the photoresist mold (Fig. 6(d)) as the ferromagnetic core. Table 1 summarizes the electroplating conditions and gives the electrolyte concentrations. After this step the seed layer is etched without using a photolithography step (Fig. 6(e)).

The 3rd and the last polyimide layers are coated over the FeNi layer. The thickness of this layer is $3.5 \mu \mathrm{m}$, covering the FeNi layer. The polyimide is patterned after this step by dry etching with an AZ9260 photoresist mask (Fig. 6(f)). This step etches all the polyimide layers down to the AlSi layer underneath the etching region, and provides the via openings for the excitation rod and the pad openings for the sensing coils.

Table 1

The electrolyte composition.

\begin{tabular}{ll}
\hline Chemicals & Concentration (mol/l) \\
\hline $\mathrm{NiSO}_{4} \cdot 6 \mathrm{H}_{2} \mathrm{O}$ & 0.7 \\
$\mathrm{FeSO}_{4} \cdot 7 \mathrm{H}_{2} \mathrm{O}$ & 0.03 \\
$\mathrm{NiCl}_{2} \cdot 6 \mathrm{H}_{2} \mathrm{O}$ & 0.02 \\
$\mathrm{H}_{3} \mathrm{BO}_{3}$ & 0.4 \\
$\mathrm{Saccharine}$ & 0.016 \\
$\mathrm{pH}$ & $2.3 @$ room temperature \\
Temperature & $40{ }^{\circ} \mathrm{C}$ \\
\hline
\end{tabular}

The process ends with the deposition and patterning of the 4th AlSi layer (Fig. 6(g)). This layer forms the upper part of the excitation rod including the via fillings. A slightly higher thickness of $2.5 \mu \mathrm{m}$ is selected in order to ensure enough step coverage at the via regions for electrical contact.

Fig. 7(a) and (b) shows the microscope picture of the fabricated fluxgate sensors and a detail around one core edge, respectively. Fig. 7(c) is an SEM image of one of the electroplated ring cores, and Fig. 7(d) is an SEM image of a via region, showing the step coverage of the 4 th metal layer. The fabricated sensors have 12 or 18 cascaded rings, each ring having $7 \mu \mathrm{m}$ and $13 \mu \mathrm{m}$ inner and outer radiuses, respectively. The sensors are placed in $1.5 \mathrm{~mm} \times 2 \mathrm{~mm}$ chips; however the coil and core structures occupy an area smaller than $0.1 \mathrm{~mm}^{2}$.

\section{Results and discussion}

\subsection{Electroplated layer properties}

Fig. 8 shows the measured $M-H$ curve of a $1.25 \mathrm{~mm}$ $\times 35 \mathrm{~mm} \times 2 \mu \mathrm{m}$ FeNi layer which is placed over the wafer as a test

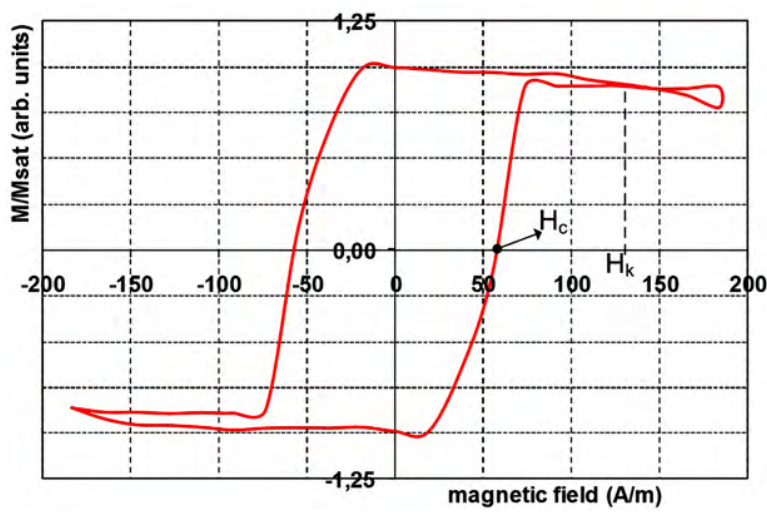

Fig. 8. The measured $M-H$ curve of the electroplated FeNi layer. 


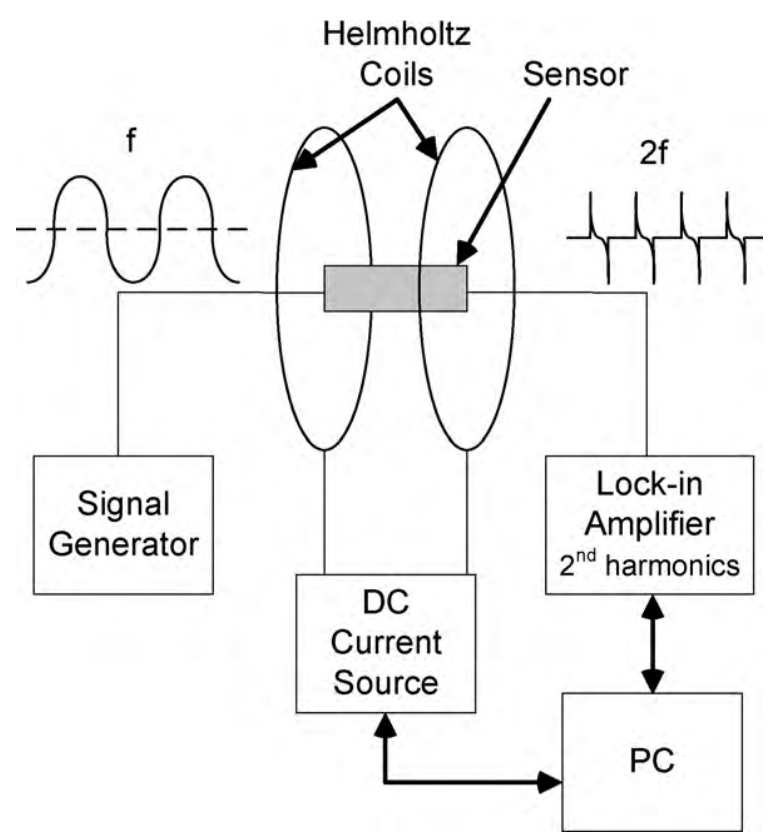

Fig. 9. The sensor test setup.

structure, and hence electroplated at the same time with the sensor structures. The $M-H$ curve is measured by using the induction method. The measured coercive field $\left(H_{\mathrm{c}}\right)$ and saturation field $\left(H_{\mathrm{k}}\right)$ values are $60 \mathrm{~A} / \mathrm{m}$ and $130 \mathrm{~A} / \mathrm{m}$, respectively.

\subsection{Test setup}

The signal conditioning of the sensor is done by external electronics. Fig. 9 shows the schematic view of the test setup. A sinusoidal excitation current produced by a signal generator is fed to the excitation rod. The Helmholtz coils produce the external magnetic field to be measured. The 2nd harmonic component of the induced voltage across the pick-up coils is measured with a lock-in amplifier synchronized with the signal generator.

\subsection{Sensor performance}

Fig. 10 shows the response of an 18-ring sensor for two different excitation current peak values at $100 \mathrm{kHz}$ frequency. The sensor has $\mathrm{a} \pm 300 \mu \mathrm{T}$ linear operation range with $198 \mu \mathrm{V} / \mathrm{mT}$ sensitivity for a sinusoidal excitation current having $170 \mathrm{~mA}$ peak value.

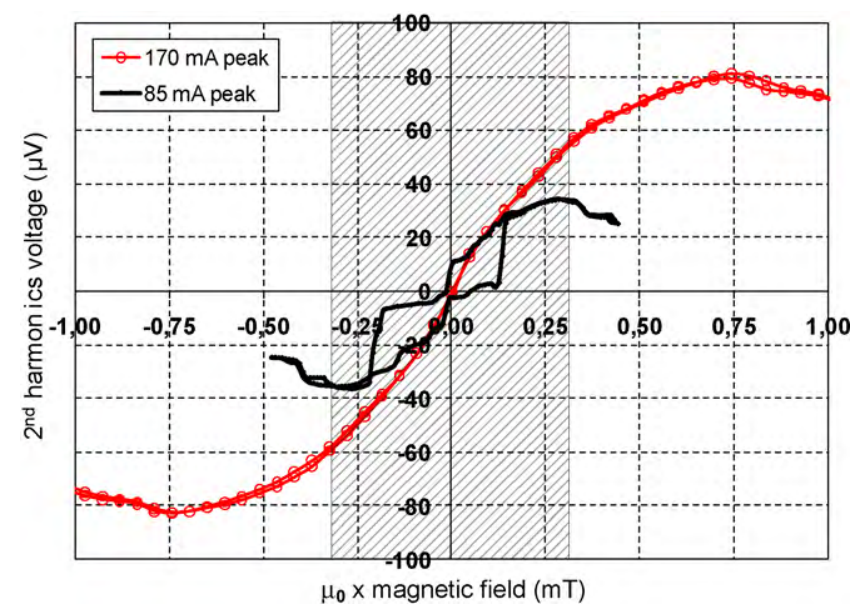

Fig. 10. The response of a sensor for two different excitation current peak values at $100 \mathrm{kHz}$ frequency.

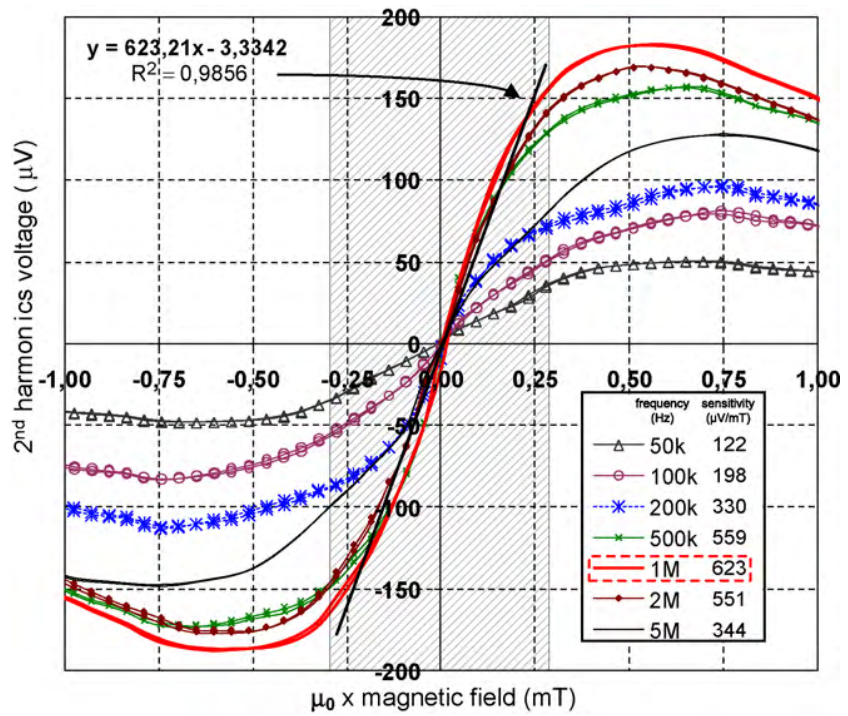

Fig. 11. The response of the sensor for different excitation current frequency values, with highest sensitivity at $1 \mathrm{MHz}$ excitation frequency.

On the other hand, the response of the sensor with a current having $85 \mathrm{~mA}$ peak value has a wide hysteresis loop, showing that the sensor cannot be completely saturated with this current value. According to the measured DC $M-H$ curve of the electroplated FeNi layer and the simulations, an excitation current with less than $50 \mathrm{~mA}$ peak value should be enough for deep saturation of the sensor core with the corresponding dimensions. One reason of the disagreement with the simulation and test results may be the higher $H_{\mathrm{k}}$ value of the AC hysteresis curve of the FeNi layer. Another reason may be a short circuit between the core and the excitation rod, which changes the flow direction of the applied current. The excitation current can be reduced to the values predicted by the simulations by slightly modifying the fabrication process flow so that the core and the excitation rod are perfectly isolated from each other. The excitation current can further be reduced by using a ferromagnetic layer having lower $H_{\mathrm{c}}$ and $H_{\mathrm{k}}$ values.

Fig. 11 shows the response of the sensor for different excitation current frequency values with $170 \mathrm{~mA}$ peak sinusoidal excitation current. The sensitivity of the sensor increases with the frequency up to $1 \mathrm{MHz}$ and reaches to $623 \mu \mathrm{V} / \mathrm{mT}$. For higher frequencies, skin effect becomes dominant and the relative permeability of the electroplated layer decreases. Hence, a decrease in the sensitivity of the sensor is observed for higher excitation frequencies. It should be noted that the sensitivity values presented in Fig. 11 are calculated for $\pm 300 \mu \mathrm{T}$ linear operation range.

Fig. 12 presents the equivalent magnetic noise spectrum of the sensor for $170 \mathrm{~mA}$-peak sinusoidal excitation at $1 \mathrm{MHz}$ frequency, under an applied external magnetic field of $90 \mu \mathrm{T}$. The measured magnetic noise of the sensor is $270 \mathrm{nT} / \sqrt{ } \mathrm{Hz}$ at $1 \mathrm{~Hz}$ and the RMS noise is $461 \mathrm{nT}$ within a $1-10 \mathrm{~Hz}$ frequency bandwidth. A peak around $50 \mathrm{~Hz}$ is visible in the frequency spectrum since no magnetic shielding is used during the measurements. The lock-in amplifier is used as a demodulator and connected to a spectrum analyzer. The time constant of the lock-in amplifier is kept at the minimum value of $100 \mu$ s so that the averaging of the lock-in amplifier itself does not distort the low-frequency part of the noise spectrum. The measured voltage noise is translated to magnetic noise through the sensor sensitivity. The measured noise and linear operation range values correspond to 10-bit resolution for the presented sensor within a $1-10 \mathrm{~Hz}$ bandwidth.

Fig. 13(a-c) shows the screen captures of the oscilloscope waveform of the excitation signal and the induced voltage across the 


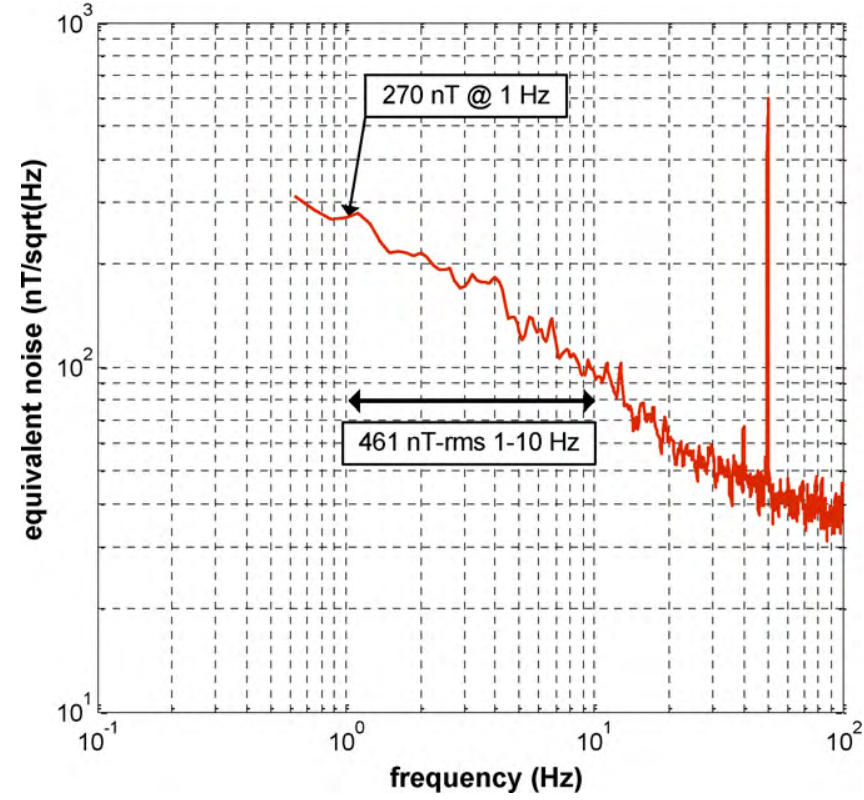

Fig. 12. The equivalent magnetic noise spectrum of the sensor for $170-\mathrm{mA}$ peak sinusoidal excitation at $1 \mathrm{MHz}$ frequency, under an applied external magnetic field of $90 \mu \mathrm{T}$.

sensing coils. The upper trace is the excitation waveform and the lower trace is the sensing coil signal at (a) $0 \mathrm{~T}$, (b) $-100 \mu \mathrm{T}$, and (c) $100 \mu \mathrm{T}$ external magnetic field density values. The excitation frequency is set to $500 \mathrm{kHz}$. The dominant 1st harmonic signal at the output of the sensing coils is visible in the screenshots. This may be coming from the sensing coils which are not perfectly symmetrical and from the misalignments during the fabrication steps. Still, the effect of higher order harmonics can be observed with the anti-symmetric deformation on the sinusoidal signal. In order to better visualize added induced voltage at two times the excitation frequency, the coil signal at $100 \mu \mathrm{T}$ external magnetic field density is subtracted from the signal at zero external field. Fig. 13(d) illustrates the result of this subtraction showing the waveform at $1 \mathrm{MHz}$, i.e., twice the frequency of excitation.

The effect of changing the number of rings on the linear operation range is presented by comparing two sensor structures. Fig. 14 shows the comparison of the responses of two sensors formed of

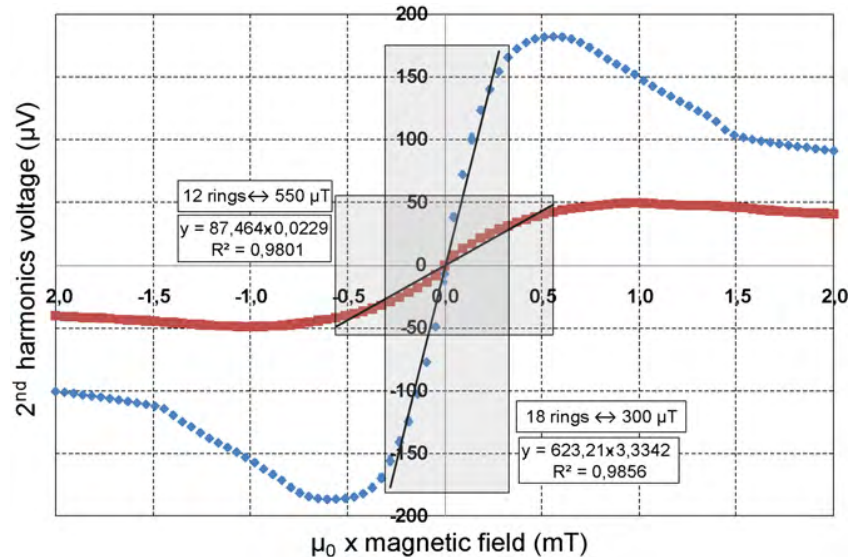

Fig. 14. The comparison of the responses of the sensors having 18 and 12 rings. Linear operation ranges of $\pm 300 \mu \mathrm{T}$ and $\pm 550 \mu \mathrm{T}$, respectively are achieved, verifying that the linear operation range can be arranged by selecting the number of rings to be used for the sensor.

12 and 18 cascaded rings. The linear operation range is $\pm 550 \mu \mathrm{T}$ for the 12 ring sensor, while that of the 18 ring sensor is $\pm 300 \mu \mathrm{T}$. The excitation current is same for both of the sensors as the single ring structures are same, verifying that the linear operation range can be arranged by selecting the number of rings to be used for the sensor. The obtained linear operation ranges are in agreement with the simulation results presented in Fig. 5. The decrease in the sensitivity for the 12-core sensor is expected up to a certain point; however, the higher difference is due to the non-uniformity in the polyimide isolation layer thickness, causing the change in the distance between the core structure and the sensing coils.

The maximum linear operation range and sensitivity is obtained with another structure composed of 4 rings and has $r_{1}, d_{2}$, and $d_{3}$ dimensions of $12 \mu \mathrm{m}, 10 \mu \mathrm{m}$, and $16 \mu \mathrm{m}$, respectively. The thickness of the FeNi core is $2 \mu \mathrm{m}$. Fig. 15 shows the response of the sensor excited at $1 \mathrm{MHz}$ with $180 \mathrm{~mA}$-peak current. The linear operation range of this sensor is $\pm 2 \mathrm{mT}$, with a sensitivity of $730 \mu \mathrm{V} / \mathrm{mT}$. This linear operation range is the widest range achieved with the microfabricated fluxgate type sensors, without using a feedback loop, to the best of our knowledge. The increase in the linear operation range with respect to the sensor with 18 rings is due to the much higher demagnetization factor of the structure. The sensi-
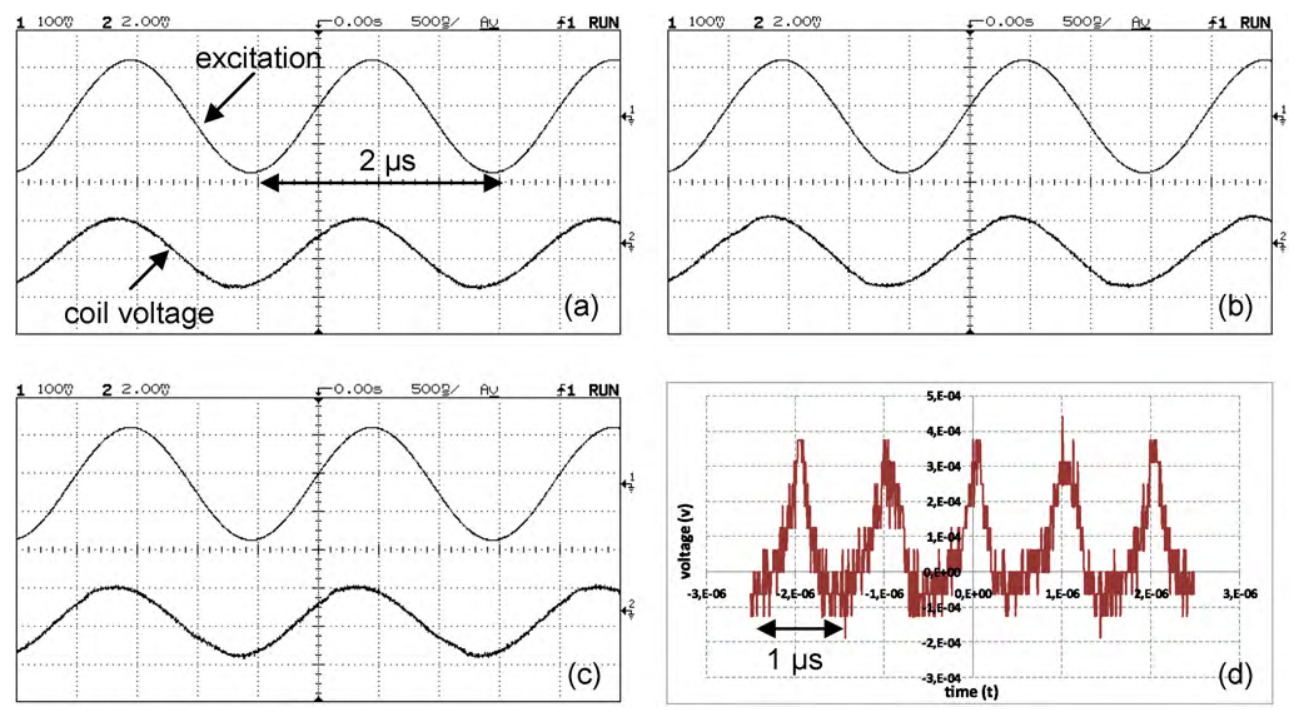

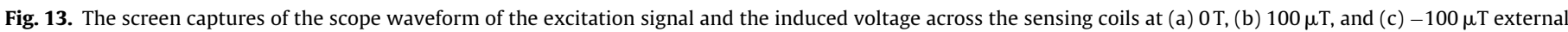
magnetic field density values, and (d) calculated induced voltage with twice the frequency of the excitation. 


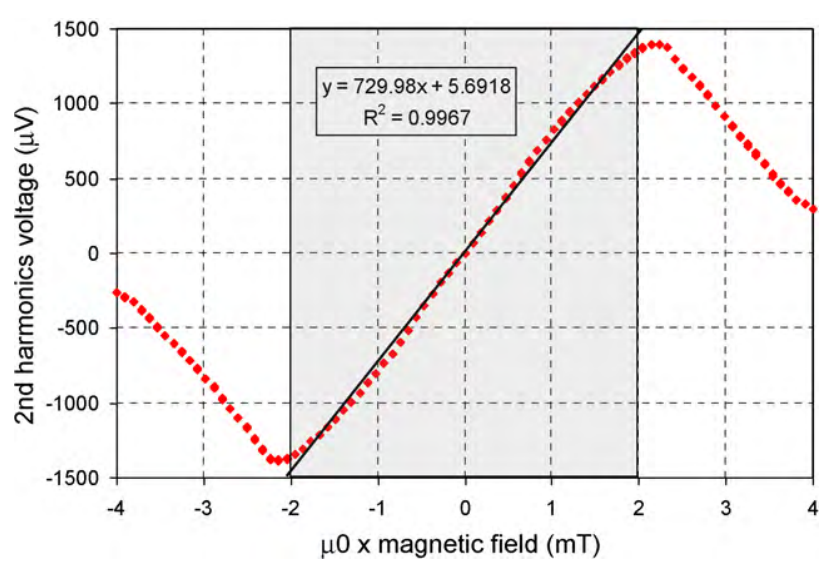

Fig. 15. The response of the sensor excited at $1 \mathrm{MHz}$ with $180 \mathrm{~mA}$ peak current reaching $\pm 2 \mathrm{mT}$ linear operation range.

tivity of the sensor is higher than that of the previous sensors because of the increased amount of magnetic material existing in the larger ring structure. Furthermore, the excitation current value that is used is an expectable value for the ferromagnetic rings having $38 \mu \mathrm{m}$ outer radius and the $M-H$ curve presented in Fig. 8 . This may show that there is no short circuit between the core and the excitation rod for this structure, and the current flows only through the excitation rod in the designed direction.

\section{Conclusion}

A new microfabricated fluxgate sensor structure having cascaded planar rings as the ferromagnetic core is presented. The closed core configuration enables uniform saturation of the ferromagnetic core and decreases the required excitation current. Furthermore, structures are microfabricated in a very small area $\left(<0.1 \mathrm{~mm}^{2}\right)$ with reduced number of via connections. The sensors consist of AlSi sputtered planar sensing coils and an excitation rod, passing through the cascaded ferromagnetic rings of electroplated FeNi. It is demonstrated by simulations and fabricated prototypes that the linear operation range of the sensor can be arranged by only changing the number of ring cores, without affecting the excitation mechanism. The sensors having 18 and 12 ring cores are characterized with $\pm 300 \mu \mathrm{T}$ and $\pm 550 \mu \mathrm{T}$ linear operation ranges, respectively.

The characterization results of the sensors show that the thin FeNi layer enables operation at excitation frequencies as high as $1 \mathrm{MHz}$. A short circuit between the ring cores and the excitation rod is pointed in the microfabricated prototypes, increasing the peak value of the required excitation current. The excitation current can be reduced by removing this short circuit problem, and the required current peak value can be reduced to the level predicted by the FEM simulations. Further improvement can be done by using a ferromagnetic layer having lower $H_{\mathrm{c}}$ and $H_{\mathrm{k}}$ values.
The mentioned short circuit problem is resolved with a larger sized 4-ring-core sensor. This sensor can be operated with $180 \mathrm{~mA}$ peak excitation current as expected. With this prototype, the achieved linear operation range is $\pm 2 \mathrm{mT}$, which is the highest reported linear operation range for microfabricated fluxgate type magnetic sensors, to the best of our knowledge.

\section{References}

[1] F. Kaluza, et al., New and future applications of fluxgate sensors, Sensors \& Actuators A 106 (2003) 48-51.

[2] L. Perez, C. Aroca, P. Sanchez, E. Lopez, M.C. Sanchez, Planar fluxgate sensor with an electrodeposited amorphous core, Sensors and Actuators A: Phsyical 109 (January (3)) (2004) 208-211.

[3] P. Ripka, Race-track fluxgate sensors, Sensors \& Actuators A 37-38 (1993) 417-421.

[4] P.M. Drljaca, P. Kejik, F. Vincent, D. Piguet, R.S. Popovic, Low-power 2-D fully integrated CMOS fluxgate magnetometer, IEEE Sensors Journal 5 (October (5)) (2005) 909-915.

[5] P. Ripka, S. Kawahito, S.O. Choi, A. Tipek, M. Ishida, Micro-fluxgate sensor with closed core, Sensors \& Actuators A 91 (2001) 65-69.

[6] P.M. Drljaca, F. Vincent, P. Kejik, R.S. Popovic, Advanced process of the magnetic core integration for the micro fluxgate magnetometer, Sensors \& Actuators A 129 (2006) 58-61.

[7] T.M. Liakopoulos, C.H. Ahn, A micro-fluxgate magnetic sensor using micromachined planar solenoid coils, Sensors and Actuators A 77 (September (1)) (1999) 66-72.

[8] H.S. Park, J.S. Hwang, W.Y. Choi, D.S. Shim, K.W. Na, S.O. Choi, Development of micro-fluxgate sensors with electroplated magnetic cores for electronic compass, Sensors and Actuators A 114 (September (2-3)) (2004) 224-229.

[9] O. Zorlu, P. Kejik, W. Teppan, Micro fluxgate sensor with cascaded planar ring cores, in: Proceedings of the Eurosensors XXIII Conference, Procedia Chemistry, vol. 1, 2009, pp. 630-633.

\section{Biographies}

0. Zorlu was born in Zonguldak, Turkey, in 1978. He received his BSc and MSc degrees in electrical and electronics engineering with honors from Middle East Technical University (METU), Ankara, Turkey, in 2000 and 2002 respectively, and his PhD degree in microtechnology from Ecole Polytechnique Federale de Lausanne (EPFL), Switzerland in 2008. He worked as a research assistant METU and EPFL between 2000 and 2008. In 2008, he joined the METU-MEMS Research and Application Center as a research fellow. His research interests include the design and fabrication of microsensors, CMOS-integrated sensor design, MEMS fabrication technologies, fluxgate type magnetic micro sensors, magnetic thin films, magnetic sensors and actuators, and MEMS-based energy harvesting.

P. Kejik received the diploma degree in 1994 and the PhD degree in 1999 at the Czech Technical University of Prague. In 1999, he joined the Institute of Microelectronics and Microsystems at the EPFL to work on the institute's circuit design and testing. His research interests include fluxgate magnetometry and micro-Hall sensors combined with mixed-signal IC design and low-noise circuit design for industrial applications. He holds five patents related to newel magnetic sensing structures and methods in the domain of contactless current measurement and angular sensing. He has authored or co-authored over 20 technical papers and he is giving a lecture devoted to recent developments in the field of smart Hall microsystems within the frame of a yearly Europractice course "Smart Sensor Systems".

W. Teppan, after some years at the Institute for Electrical Drives at the Graz University of Technology, Austria, where he specialized in current measurement, co-founded a start-up company active in the field of electrical measurement. In 1998 this company merged with LEM, where he led several development projects for LEM's former instrument division. Since he joined LEM components in 2001, his main focus is work on innovative solutions for current measurement. 